\title{
Controle com Compensação de Zona Morta de um Atuador Pneumático para Posicionamento de um Equipamento para Poda de Árvores
}

\author{
Rozimerli Raquel Milbeier Richter ${ }^{1}$, Claudio da Silva dos Santos ${ }^{2}$, \\ Antonio Carlos Valdiero ${ }^{3}$, Luiz Antonio Rasia ${ }^{4}$ \\ Departamento de Ciências Exatas e Engenharias, UNIJÚ, Panambi, RS
}

\begin{abstract}
Resumo. Este trabalho apresenta o controle de posição de um atuador pneumático com compensação da não linearidade de zona morta para uma dada aplicação em um equipamento para poda de árvores. Os resultados ilustram a importância da modelagem matemática, da identificação experimental de parâmetros e da compensação das não linearidades.
\end{abstract}

Palavras-chave. Zona morta, Controle de posição, Compensação.

\section{Introdução}

Atuadores pneumáticos são sistemas muito atrativos para diversas aplicações, em especial na robótica, porque eles têm a vantagem de baixo custo, leveza, durabilidade e são limpos quando comparados com os atuadores hidráulicos, também possuem facilidade de manutenção, têm boa relação força/tamanho e flexibilidade de instalação e, além disso, o ar comprimido está disponível na maioria das instalações industriais $[1,3]$. Os servoposicionadores pneumáticos também apresentam menor risco de contaminação ambiental e de operação em relação aos sistemas hidráulicos, visto que, se ocorrer no sistema hidráulico um vazamento de óleo, isso poderá gerar sérios danos ambientais, ainda maiores se este óleo for inflamável, destaca [1].

Em despeito dessas vantagens, sistemas de posicionamento pneumático possuem algumas características indesejáveis as quais limitam o uso destes em aplicações que requerem uma resposta precisa $[1,2]$. Estas características indesejáveis derivam da alta compressibilidade do ar [3] e das não linearidades presentes em sistemas pneumáticos, tais como o comportamento não linear da vazão mássica nos orifícios da válvula e sua zona morta [3], além do atrito nas vedações do cilindro linear. Estas características indesejáveis podem ser compensadaspor meio de estratégias de controle adequadas [2].

\footnotetext{
${ }^{1}$ rozimerlirichter@gmail.com

2 prof.claudiosant@hotmail.br

3 valdiero@unijui.edu.br

${ }^{4}$ rasia@unijui.edu.br
} 
Valdiero et al. [3] descreve o quão importante é o estudo das não linearidades presentes nos sistemas mecânicos, as quais causam limitações no desempenho do controle preciso, portanto destacando-se uma das necessidades é de identificação e compensação da zona morta a partir da observação da dinâmica do comportamento das pressões nos orifícios das válvulas. Richter et al. [1] a importância do estudo das características não lineares presentes nos atuadores pneumáticos devido à dificuldade de controle dos mesmos.

\section{Propósito}

O propósito deste trabalho é apresentar a importância da modelagem, da identificação e do projeto de uma estratégia de controle que inclua a compensação da não linearidade da zona morta em servoválvulas pneumáticas de controle direcional. Os resultados obtidos nesta pesquisa contribuíram para o projeto e construção de uma haste telescópica utilizada num equipamento pneumático de poda, conforme apresentado na Figura 1.

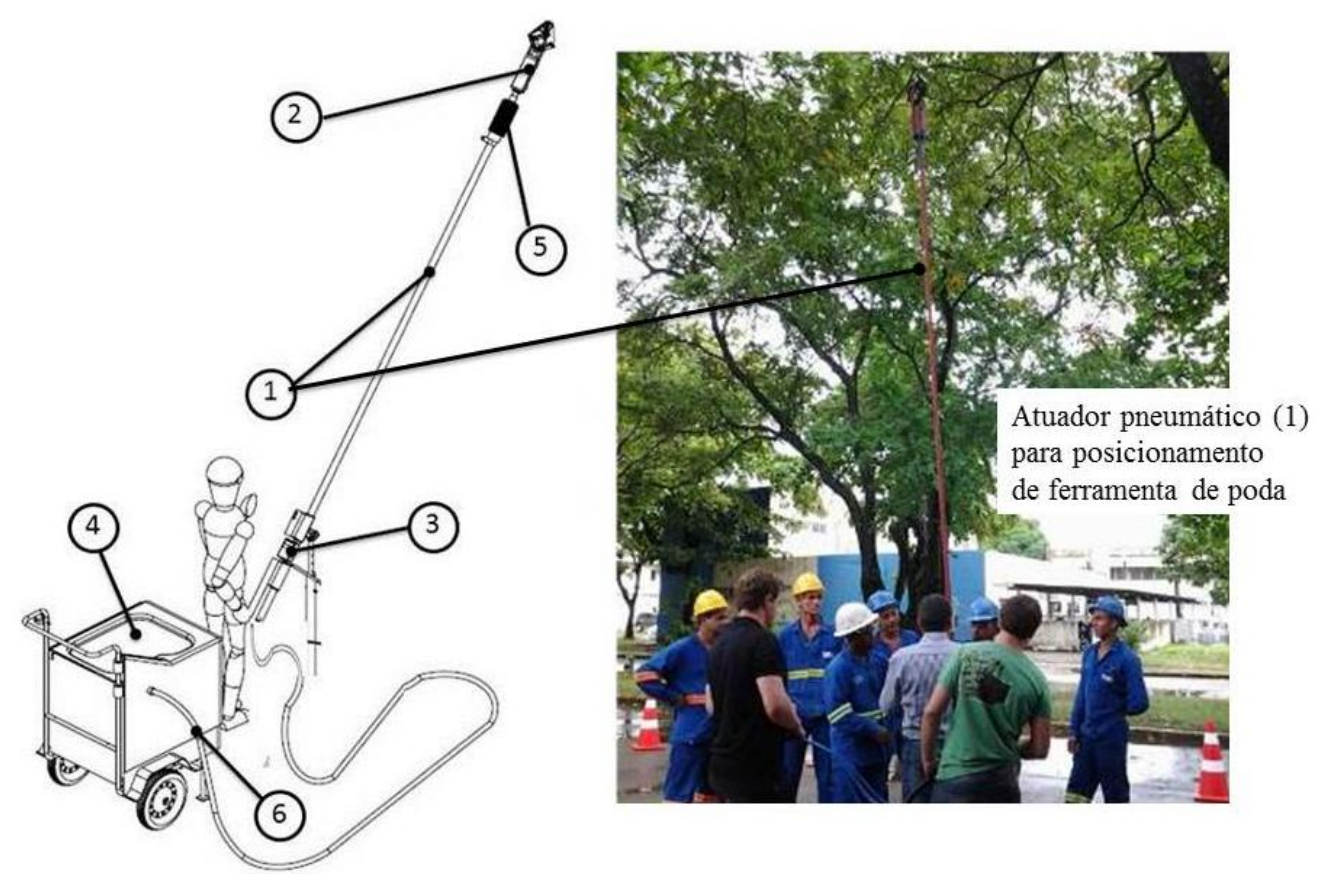

Figura 1: Aplicação do controle de um atuador pneumático com compensação de zona morta em um equipamento para poda de árvores.

Para melhor entendimento da aplicação, faz-se as indicações de seus principais componentes na Figura 1. O equipamento pneumático para poda de árvores é compreendido por um módulo de posicionamento e orientação (1), módulo de corte (2), comando e geogerenciamento (3), módulo de potência (4), sanfona isolante (5) e (6) mangueira corrugada de proteção. É necessário levar em consideração a não linearidade da zona morta, que neste trabalho é considerada como sendo a sobreposição do ressalto do carretel da servoválvula em relação ao orifício de passagem do ar sob pressão, já que a largura do ressalto do carretel é maior que a largura do orifício. Valdiero et al.[3] destaca que este tipo de imperfeição é bastante comum em sistemas mecânicos, principalmente em servoválvulas. A presença da zona morta nas servoválvulas gera limitações significativas no desempenho de controladores por realimentação, principalmente no que diz respeito à minimização do erro 
de posicionamento e de seguimentos de trajetórias, diante disso, se faz necessário a utilização de metodologias de identificação e compensação dessa não linearidade. Na seção seguinte descreve-se o modelo matemático da não linearidade de zona morta.

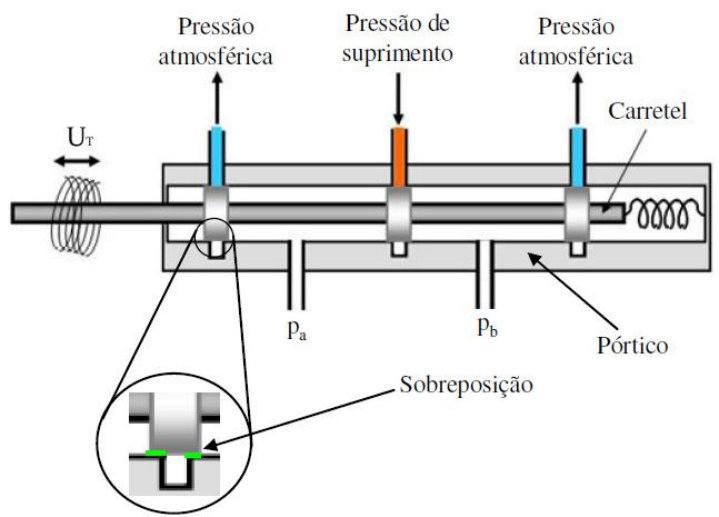

Figura 2: Desenho esquemático do corte de uma servoválvula direcional com seus principais elementos mecânicos.

\section{Modelagem Matemática da Zona Morta}

A zona morta é uma relação estática de entrada e saída, em que para uma faixa de domínio não há resposta, ou seja, a saída é nula, apresenta-se um modelo genérico para a zona morta em servoválvulas de controle direcional baseado em Tao e Kokotovic[2] pela Eq. (1):

$$
U_{z m}(t)=\left\{\begin{array}{cc}
m d\left(U_{T}(t)-z m d\right) & \text { se } U_{T}(t) \geq z m d \\
0 & \text { se zme }<U_{T}(t)<z m d \\
m e\left(U_{T}(t)-z m e\right) & \text { se } U_{T}(t) \leq z m e
\end{array}\right.
$$

onde $U_{T}(t)$ é o sinal de entrada, $U_{z m}(t)$ é o valor de saída, $z m d$ é o limite direito da zona morta, $z m e$ é o limite esquerdo da zona morta, $m d$ é a inclinação direita da zona morta e $m e$ é a inclinação esquerda da zona morta. Estes valores para a zona morta foram obtidos experimentalmente e descritos na seção seguinte.

A Figura 3 mostra a representação gráfica do trecho de zona morta do sinal de entrada $U=U_{T}$ em relação ao sinal de saída $U_{z m}$.

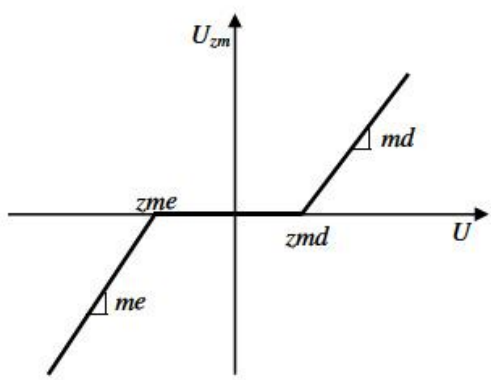

Figura 3: Representação gráfica da não linearidade de zona morta. 
A servoválvula proporcional utilizada para controlar o escoamento de ar comprimido para as câmaras do cilindro pneumático (haste telescópica do equipamento de poda) mostrado na Figura 4. Sendo que o deslocamento do carretel da servoválvula é proporcional ao sinal de controle $U_{T}$ abre os orifícios de controle para que uma câmara do cilindro linear seja ligada a linha de pressão de suprimento e a outra seja ligada à pressão atmosférica $\left(p_{a t m}\right)$. Desta forma, produzindo uma diferença de pressão nas câmaras do cilindro linear, dando origem a uma força resultante movendo a massa $M$ acoplada a haste deste cilindro, em um deslocamento no sentido positivo ou negativo $y$, o qual deve ser controlado.

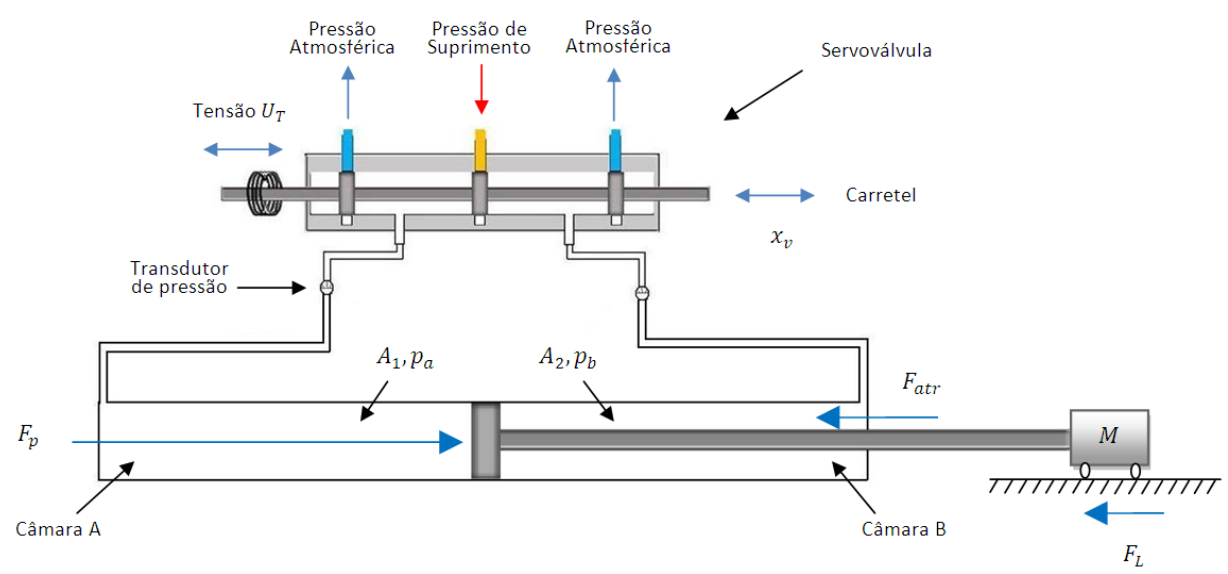

Figura 4: Desenho esquemático de um servoposicionador pneumático linear.

\section{Métodos}

Para a identificação dos parâmetros do modelo da zona morta e a implementação da estratégia de controle, utiliza-se a ferramenta Simulink do software MatLab. Os testes experimentais são realizados na infraestrutura disponível na UNIJUÍ Câmpus Panambi, no Núcleo de Inovação e Mecanização da Poda (NIMeP), em uma bancada de testes experimentais. A Figura 5 mostra um desenho esquemático da bancada de testes composta por um servoposicionador pneumático linear e um sistema de instrumentação eletrônica dSPACE.

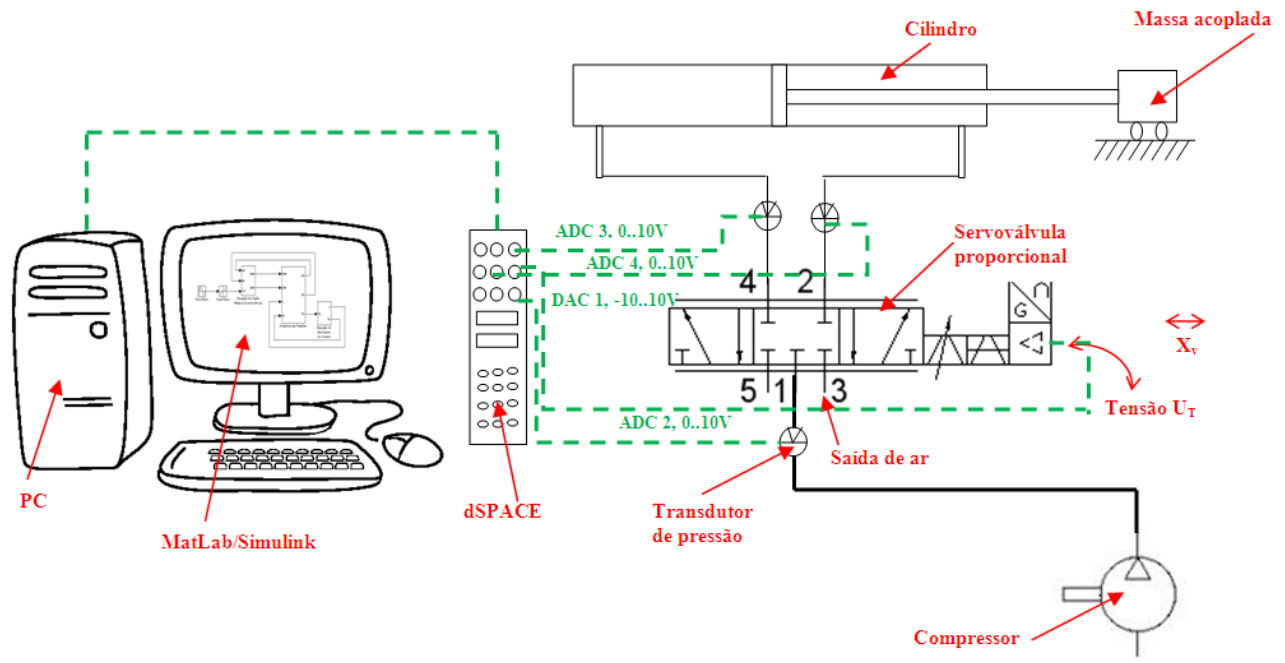

Figura 5: Bancada de testes experimentais utilizada no controle com compensação de zona morta. 
A identificação convencional da zona morta é realizada a partir de testes experimentais realizados, utilizando-se uma metodologia de baixo custo proposta por Valdiero [3] que utiliza apenas transdutores de pressão.

Para identificar a região de zona morta é realizado um ensaio em malha aberta com um sinal de controle senoidal lento, com amplitude de $10 \mathrm{~V}$ e período de 100 segundos para completar um ciclo, dado pela Eq. (2).

$$
U_{T}(t)=10 \operatorname{sen}\left(\frac{2 \pi}{100} t\right)
$$

Como resultado tem-se dois trechos para este sinal de controle. No primeiro, o sinal de controle $U_{T}$ varia de $10 \mathrm{~V}$ a $-10 \mathrm{~V}$, onde é analisada a dinâmica da pressão na câmara B do cilindro, possibilitando a identificação do limite esquerdo da zona morta, conforme apresentado nos resultados da seção seguinte.

\section{Resultados}

Os resultados obtidos compreendem a identificação experimental dos parâmetros da zona morta, a definição de uma estratégia para sua compensação no controle e os resultados de testes experimentais de controle em malha fechada.

A partir da metodologia descrita na seção anterior e com maiores detalhes em [1], obtém-se o comportamento das pressões em função do sinal de controle, pois a partir do momento em que o carretel começa a bloquear os orifícios de passagem do ar, neste trecho o sinal está próximo a sua origem resultando em vazamentos significativos. A Figura 6 mostra à dinâmica das pressões em ambas as câmaras, gerada pelo sinal de controle senoidal aplicado a servoválvula.

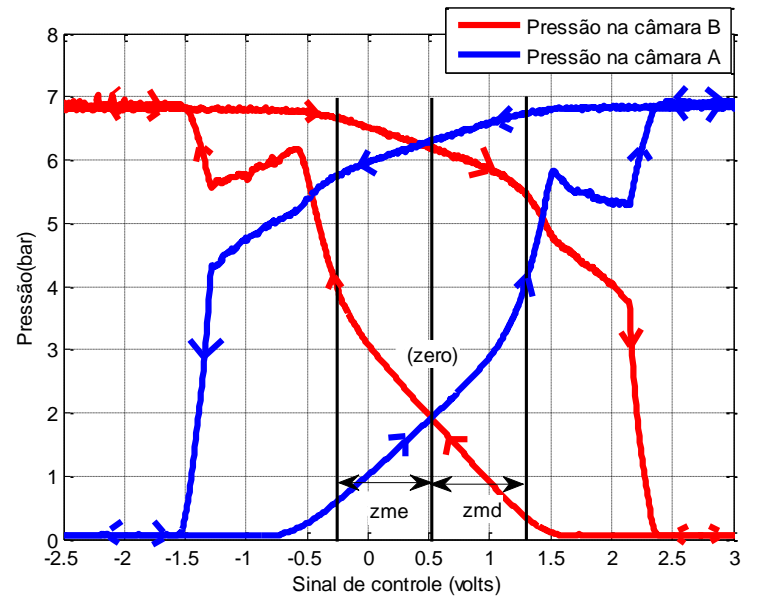

Figura 6: Gráfico da dinâmica das pressões, centro da servoválvula e identificação dos limites da zona morta.

Ao observar a dinâmica das pressões em ambas as câmaras, percebe-se que os trechos não são simétricos em relação à origem do sinal de controle, ou seja, o centro da servoválvula não se encontra na origem do sinal de controle.

Observando o gráfico expresso pela Figura 6, tem-se o zero, determinado pelo ponto 
médio dos trechos de variação das pressões causadas pelos vazamentos internos. Enfim, a partir da análise e determinação, estes valores da não linearidade da servoválvula em relação à origem do sinal de controle são (no zero $=0,52 \mathrm{~V}$ ) e tem-se $z m d=z m e=0,78 \mathrm{~V}$. Os valores para a inclinação direita e esquerda da zona morta são iguais e unitários $(m d=$ $m e=1)$.

Como estratégia para a compensação da não linearidade da zona morta em servoválvulas, utiliza-se o modelo matemático da sua inversa na saída do controlador, com uma suavização da pela Eq. (3).

$$
U_{c z m}(t)=\left\{\begin{array}{cc}
\frac{U_{d}(t)}{m d}+z m d & \text { se } U_{d}(t) \geq l c \\
\frac{U_{d}(t)}{m e}-|z m e| & \text { se } U_{d}(t) \leq-|l c| \\
\left(\frac{z m d+l c / m d}{l c}\right) U_{d}(t) & \text { se } 0 \leq U_{d}(t)<l c \\
\left(\frac{|z m e|+|l c| / m e}{|l c|}\right) U_{d}(t) & \text { se }-|l c| \leq U_{d}(t)<0
\end{array}\right.
$$

onde, $U_{d}(t)$ é a entrada de sinal de controle desejável sem a existência da zona morta, $U_{c z m}$ é a saída de sinal compensado, $m d$ e $m e$ são as inclinações que representam a proporcionalidade entre a entrada e a saída, lc é a largura de suavização utilizada na compensação e define a região de suavização linear, esta é regulada de acordo com o ruído do sinal para não desestabilizar o sistema, sendo que quanto maior a amplitude do ruído no sinal, maior deverá ser o $l c$ e vice-versa. A Figura 7 mostra a representação gráfica da inversa da zona morta suavizada.

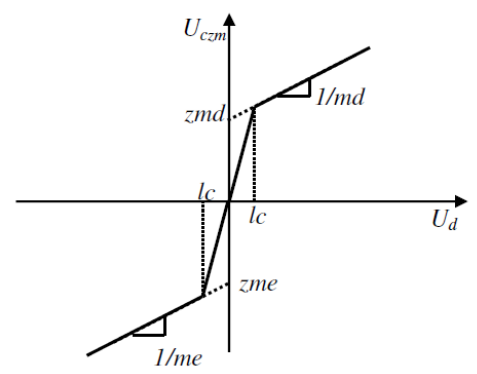

Figura 7: Representação gráfica da inversa da não linearidade de zona morta com trechos de suavização próximos a origem.

Os testes experimentais foram realizados utilizando um controlador proporcional com ganho $k_{p}=70$ como testemunha com e sem a compensação de zona morta. A Figura 8 ilustra os resultados dos testes experimentais sem e com a compensação de zona morta. 

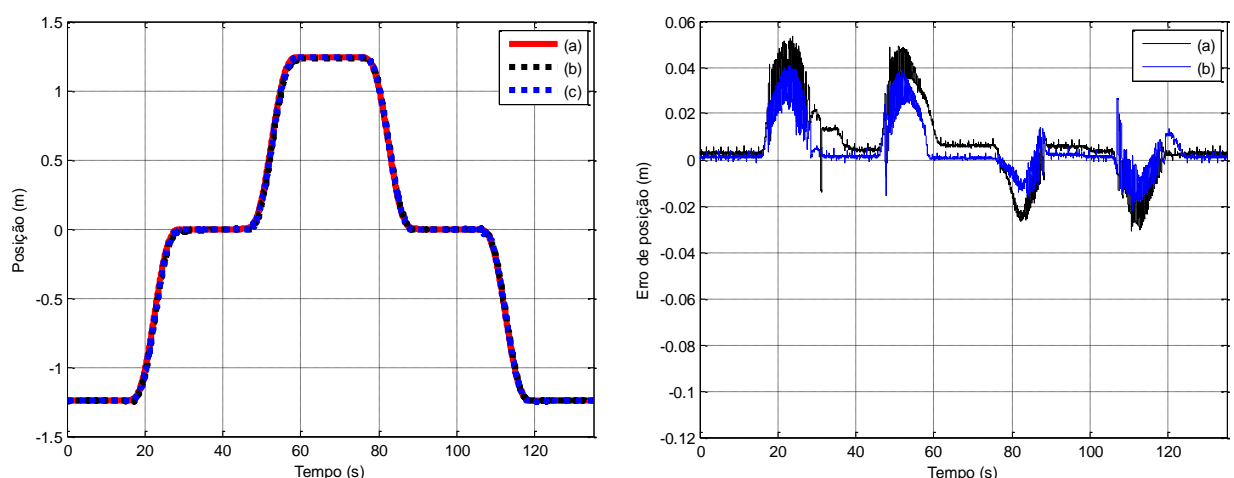

Figura 8: Gráfico da esquerda: (a) trajetória polinomial desejada, (b) seguimento sem compensação de zona morta e (c) com zona morta compensada; gráfico da direita: erro do controle de seguimento (a) sem compensar a zona morta e (b) com zona morta compensada.

\section{Conclusões}

A identificação dos parâmetros da não linearidade de zona morta da servoválvula, através da análise das pressões nos orifícios de saída, juntamente com a aplicação do esquema de compensação através da inversa fixa de seu modelo parametrizado, mostrou-se bastante eficiente. A partir da análise dos resultados, conclui-se que a compensação da zona morta é muito importante e deve ser feita para minimizar os erros de seguimento de trajetória de uma haste telescópica utilizada num equipamento pneumático de poda.

\section{Agradecimentos}

Os autores agradecem a UNIJUÍ pelo incentivo e também à CELPE (Companhia Energética de Pernambuco) pelo apoio financeiro no âmbito Programa P\&D da ANEEL (código ANEEL PD-0043-0311/2011).

\section{Referências}

[1] R. R. M. Richter, C. V. Zamberlan, A. C. Valdiero and L. A. Rasia, Trajectory Tracking Control of a Pneumatic Actuator Special, Int. J. of Computer Science and Applications (Online), (2014).

[2] G. Tao and Kokotovic, Adaptive Control of Systems with Actuators and Sensors Nonlinearities. New York: John Wiley\& Sons, (1996).

[3] A. C. Valdiero, C. S. Ritter, C. F. Rios and M. Rafikov, NonLinear Mathematical Modeling in Pneumatic Servo Position Applications, Mathematical Problems in Engineering (Online), v. 2011, p. 1-16, (2011). 